\title{
Predictions for the observation of KRb spectra under cold conditions
}

\author{
Robert Beuc $^{1}$, Mladen Movre ${ }^{1}$, Ticijana Ban ${ }^{1}$, Goran Pichler ${ }^{1}$, \\ Mireille Aymar ${ }^{2}$, Olivier Dulieu ${ }^{2}$ and Wolfgang E Ernst ${ }^{3}$ \\ ${ }^{1}$ Institute of Physics, Bijenička cesta 46, HR-10000 Zagreb, Croatia \\ 2 Laboratoire Aimé Cotton, CNRS, Bâtiment 505, Campus d'Orsay, 91405 Orsay Cedex, France \\ ${ }^{3}$ Institute of Experimental Physics, Graz University of Technology, Petersgasse 16, \\ A-8010 Graz, Austria
}

Received 28 April 2006, in final form 28 July 2006

Published 25 September 2006

Online at stacks.iop.org/JPhysB/39/S1191

\begin{abstract}
Quantum-mechanical simulations of the excitation spectra of KRb from the lowest vibrational level of the lowest triplet and singlet electronic states have been performed using recently calculated interaction potential curves and corresponding transition dipole moments. The obtained spectra can be used for a comparison with experimental absorption spectra of KRb molecules produced in their vibronic ground state or attached to cold helium droplets. In addition, we compare the semiclassically simulated spectra with absorption measurements in dense $\mathrm{K}-\mathrm{Rb}$ vapour at high temperatures, which helped us to identify three diffuse bands as $1{ }^{3} \Sigma^{+}-3{ }^{3} \Pi, 1{ }^{3} \Sigma^{+}-4{ }^{3} \Pi$ and $1{ }^{1} \Sigma^{+}-4{ }^{1} \Sigma^{+}$ transitions. The first may be observable in an excitation spectrum of $\mathrm{KRb}$ dimer formed on cold helium droplets.
\end{abstract}

\section{Introduction}

Spectra of mixed alkali molecules were studied long ago by Bevan [1] and Walter and Barratt [2] but only very recently obtained renewed interest and became the subject of new calculations and experimental investigations because of technical advances in the research of cold molecules. Alkali dimers can be formed in atomic traps [3] and dimers made from mixed species could be manipulated and slowed down because of their permanent dipole moments [4].

Recently, KRb molecules were detected under ultracold conditions [5] and laser spectroscopic experiments were carried out for $\mathrm{KRb}$ molecules in magneto-optical traps with potassium-rubidium mixtures $[6,7]$ where only near-dissociation spectra have been observed, probing the long-range parts of interaction potentials. Besides these recent trap measurements, cold helium droplets loaded with potassium and rubidium were investigated in the laboratory at Graz University of Technology and the predictions of this paper were intended to provide a guideline for the assignment of spectra. In fact, the $2{ }^{3} \Pi-1{ }^{3} \Sigma^{+}$transition was identified both in absorption and emission [8]. 
Potential energy curves for KRb molecules are known both with and without spin-orbit interaction being taken into account [9, 10]. Park et al [9] and Kotochigova et al [11] have also calculated transition dipole moments between lowest states and excited states. For the five singlet states observed $\left(1^{1} \Sigma^{+}, 3{ }^{1} \Sigma^{+} ; 1{ }^{1} \Pi, 2{ }^{1} \Pi\right.$ and $3{ }^{1} \Pi$; here and throughout the paper the states of given symmetry are labelled in order of increasing energy), the agreement between calculated [10] and experimental molecular constants was good with deviations of term values (electronic energies) $T_{\mathrm{e}}$, equilibrium distances $R_{\mathrm{e}}$ and vibrational frequencies $\omega_{\mathrm{e}}$ being $\Delta T_{\mathrm{e}}<$ $140 \mathrm{~cm}^{-1}, \Delta R_{\mathrm{e}}<0.08 \AA$ and $\Delta \omega_{\mathrm{e}}<6 \mathrm{~cm}^{-1}$, respectively. The theoretical results [9] are of similar overall quality with highest $\Delta T_{\mathrm{e}} \approx 230 \mathrm{~cm}^{-1}$ for the $3{ }^{1} \Pi$ state. Later, the molecular constants for two triplet states were experimentally determined: Amiot [12] measured the 2 ${ }^{3} \Sigma^{+}$state and Lee et al $[13,14]$, the $3{ }^{3} \Sigma^{+}$state. In addition, Lee et al [13] observed an electric quadrupole $1^{1} \Delta-1^{1} \Sigma^{+}$transition in a pulsed molecular beam and obtained molecular constants for the upper state.

For most alkali molecules, transitions between singlet electronic states are better known from spectroscopy than those involving triplet states. The lowest triplet states are only weakly bound and thus unstable in gases at elevated temperatures. In the gas phase triplet transition of alkali dimers are usually observed in the form of diffuse and satellite bands, because the lowest $1^{3} \Sigma_{\mathrm{u}}^{+}$electronic state is weakly bound, and therefore negligibly populated at thermal energies. Diffuse bands of KRb have been known for a long time [15], but their interpretation in terms of potential curves is still missing. Only the so-called triplet satellite bands of KRb have been recently interpreted [16]. On the other hand, triplet states are produced in magneto-optical traps. Also, when molecules are formed on helium nanodroplets at $0.4 \mathrm{~K}$, the weakly bound triplet dimers are observed more frequently than the singlets, because their binding energy is smaller. The binding energy is dissipated into the helium droplet and it leads to helium evaporation. Singlets have about 20 times the binding energy of triplets and there is much less chance of a droplet surviving the corresponding evaporation loss [17].

In our previous applications of potential curves from $a b$ initio calculations, it turned out to be helpful to inspect the complex manifold of potentials in both Hund's case (a) and Hund's case (c). Hund's case (a) brings about a much simpler picture of energy potentials, whereas Hund's case (c) which includes spin-orbit interaction comprises a larger number of potential curves, of which more pairs, having the same quantum-mechanical symmetry, undergo avoided crossings.

Our present simulations use Hund's case (a) potential curves and furthermore only the lowest vibrational levels of the lowest triplet and singlet electronic states were assumed to be populated because we are interested in low-temperature spectra. Interaction potentials and transition dipole moments (some of them not previously available) are presented from recent calculations in which a standard quantum chemistry approach based on pseudopotentials for atomic core representation, Gaussian basis sets and effective terms for core polarization effects, was used, as described in [18].

Besides the production of the lowest triplet state of an alkali dimer in a magneto-optic trap or on a cold helium droplet, there are other types of experiments in which absorption from the lowest triplet state is observed. Whereas the typical low-density gas-phase environment of high-resolution molecular spectroscopy experiments favours equilibrium conditions with near $100 \%$ singlet ground-state population, a high-temperature environment together with considerable pressure provides equilibrium with both lowest singlet and triplet populations. Such conditions have been used in the past in order to search for the so-called diffuse bands [16]. In this case, broad absorption features partly overlap and the assignment is not always straightforward. This identification is particularly difficult for heteronuclear dimers that require the presence of two alkali species. In Zagreb, new experiments with rubidium and 
potassium in a high-temperature cell were performed. The semiclassically calculated KRb spectra were compared with the absorption features observed in hot dense $\mathrm{K}-\mathrm{Rb}$ vapour and two triplet and one singlet diffuse bands have been identified. This comparison provides an additional opportunity to estimate the accuracy of the difference potentials around the initial state potential minimum and contribute to the further analysis of our predictions of triplet bands under cold conditions.

\section{Results and discussion}

\subsection{Potentials and dipole moments}

We performed new quantum chemistry calculations of electronic properties of heteronuclear alkali dimers [18], based on a pseudo-potential representation of atomic cores including core polarization effects, and extended Gaussian basis sets. We briefly recall the calculation procedure, which is well documented in many publications. We used an automatic procedure based on the CIPSI package [19] developed by the 'Laboratoire de Physique Quantique de Toulouse (France)'. The atomic cores are described by the pseudopotentials of Durand and Barthelat [20], core polarization is taken into account through an $l$-dependent effective core polarization potential [21], and we used the same Gaussian basis sets as Rousseau et al [10]. Also, care was taken to include correctly the core-core interaction potentials. Our results for the Hund's case (a) interaction potentials are therefore of the same overall quality as in [10].

New results for transition dipole moments which were not previously available, involving many states in all molecular symmetries have also been computed. It is not easy to estimate the accuracy of the current results. The calculated values for the permanent dipole moments of the $1{ }^{1} \Sigma^{+}$and $1{ }^{3} \Sigma^{+}$states (traditionally denoted by X and a, respectively) of KRb obtained by using quite different theoretical approaches [9, 11, 18] have already been compared in [18] and quite large differences were observed but not completely understood. However, the good agreement with available experimental results on other systems obtained in [18] was the firm basis to believe that the values given for the $\mathrm{KRb}$ permanent dipole moments are quite accurate. We believe that the same is true for the present transition dipole moments.

Relevant potential energy curves from our $a b$ initio calculations are shown in figures 1(a) and (b) for triplet and singlet manifolds, respectively. Narrow Franck-Condon windows around the equilibrium internuclear distance of the lowest singlet and triplet states are indicated by vertical bars. The prominent avoided crossing between $3{ }^{3} \Sigma^{+}$and $4{ }^{3} \Sigma^{+}$can be readily seen. There is another avoided crossing of the $3{ }^{3} \Pi$ and $4{ }^{3} \Pi$ states. The avoided crossing of the weakly coupled $1^{1} \Pi$ and $2{ }^{1} \Pi$ 'twin' states has already been discussed by Leininger et al [22]. Most transitions within the indicated Frank-Condon windows are of bound-bound character, but one can also expect bound-free excitation to the $2^{3} \Pi$ and $3{ }^{3} \Sigma^{+}$ potentials.

The relevant electric dipole transition moments are shown in figures 2(a)-(d). Pairs of transition dipole moments to the potentials exhibiting the avoided crossings $\left(1^{3} \Sigma^{+}-3{ }^{3} \Pi\right.$ and $1^{3} \Sigma^{+}-4{ }^{3} \Pi$ in figure $2(\mathrm{c})$ and $1{ }^{1} \Sigma^{+}-1{ }^{1} \Pi$ and $1{ }^{1} \Sigma^{+}-2{ }^{1} \Pi$ in figure $\left.2(\mathrm{~d})\right)$ are clearly seen to change strongly in the vicinity of the crossing point, whereas the shapes of the $1{ }^{3} \Sigma^{+}-3^{3} \Sigma^{+}$ and $1{ }^{3} \Sigma^{+}-4{ }^{3} \Sigma^{+}$curves in figure 2(a) reflect additional interaction with the $5^{3} \Sigma^{+}$state which is mixed in.

\subsection{Absorption bands under cold conditions}

Energies and wavefunctions for vibrational levels were determined by using the Fourier Grid Hamiltonian (FGH) method [23]. The potential energy operator is local in the coordinate 


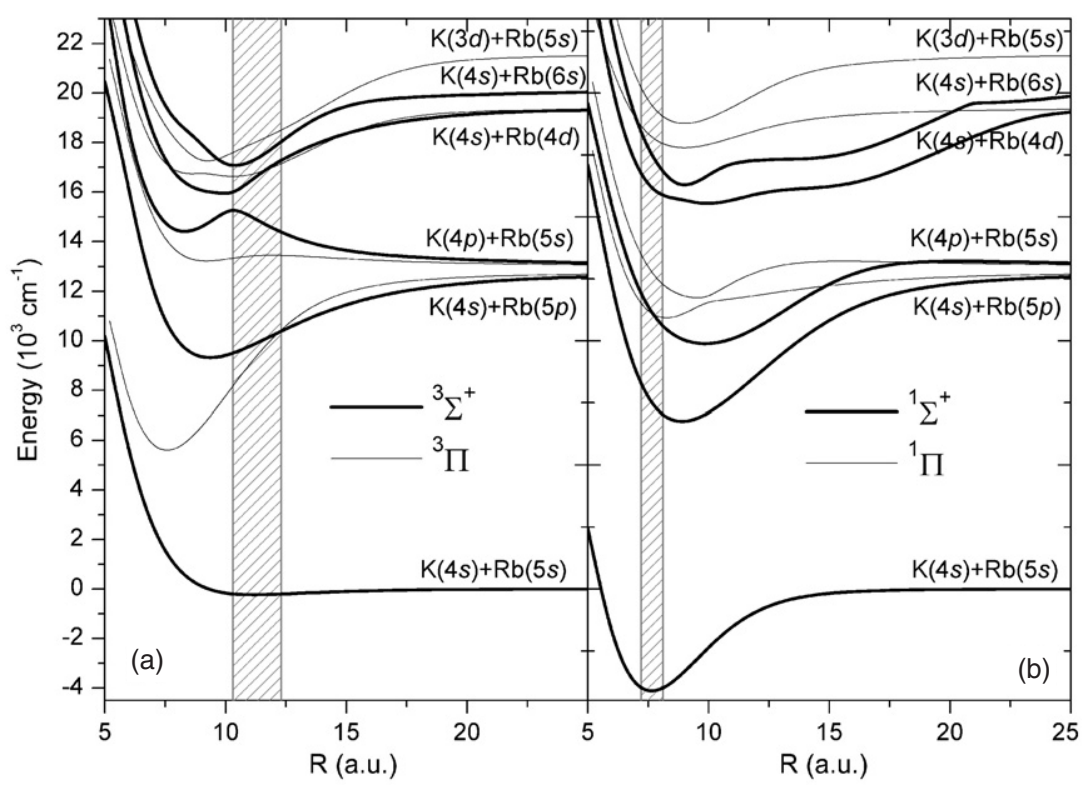

Figure 1. Potential energy curves from present ab initio calculations: (a) triplet and (b) singlet states. Narrow Frank-Condon windows around the equilibrium internuclear distance of the lowest singlet and triplet states are indicated by vertical bars.

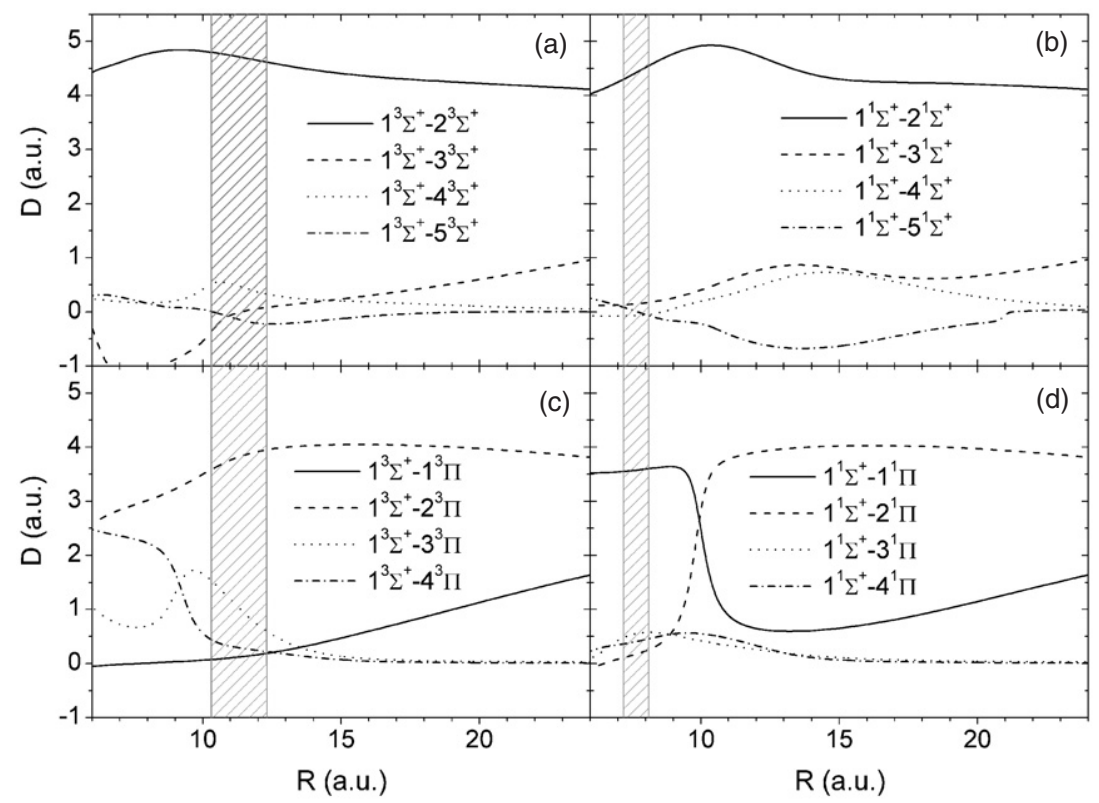

Figure 2. Electric dipole transition moments from present ab initio calculations: (a) $1^{3} \Sigma^{+}-N^{3} \Sigma^{+}$ $(N=2,3,4,5)$; (b) $1{ }^{1} \Sigma^{+}-N^{1} \Sigma^{+}(N=2,3,4,5)$; (c) $1^{3} \Sigma^{+}-N^{3} \Pi(N=1,2,3,4)$ and (d) $1{ }^{1} \Sigma^{+}-N$ ${ }^{1} \Pi(N=1,2,3,4)$. The states are labelled in order of increasing energy. Narrow Frank-Condon windows around the equilibrium internuclear distance of the lowest singlet and triplet states are indicated by vertical bars. 


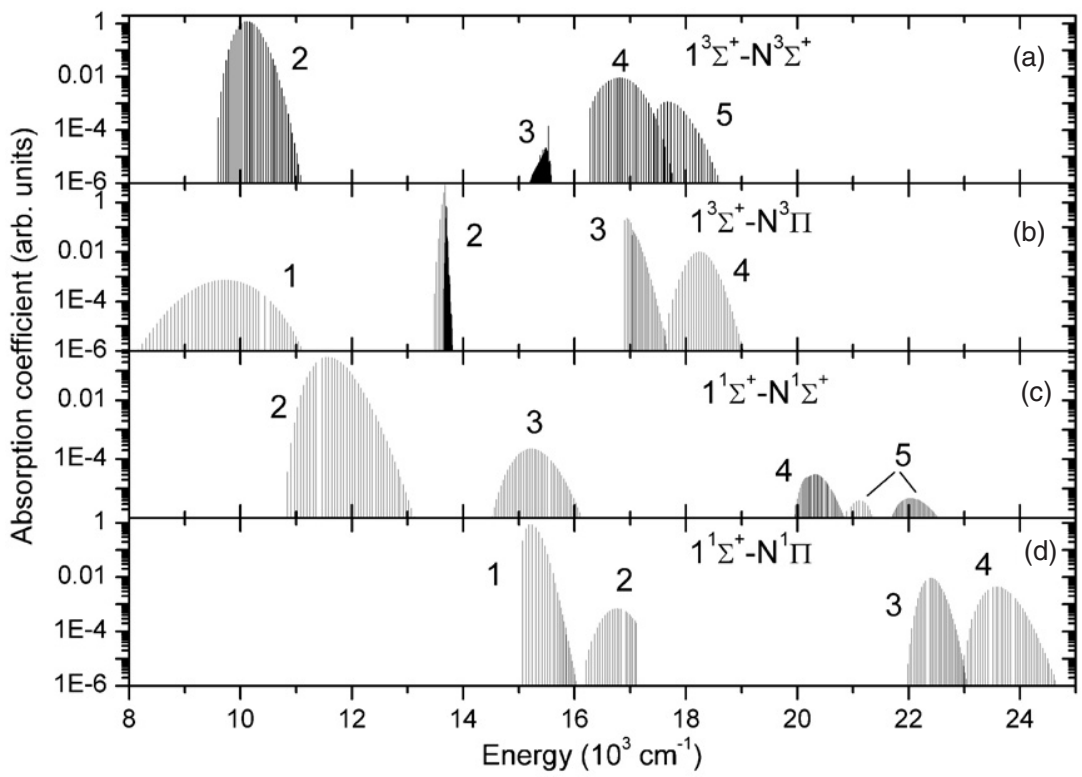

Figure 3. Calculated absorption band shapes for triplet and singlet transitions in KRb under cold conditions: (a) $1{ }^{3} \Sigma^{+}-N^{3} \Sigma^{+}(N=2,3,4,5)$; (b) $1{ }^{3} \Sigma^{+}-N^{3} \Pi(N=1,2,3,4)$; (c) $1{ }^{1} \Sigma^{+}-N^{1} \Sigma^{+}$ $(N=2,3,4,5)$; (d) $1{ }^{1} \Sigma^{+}-N^{1} \Pi(N=1,2,3,4)$. The contributions of the bound-free continua, not well resolved in figures $3(\mathrm{a})$ and (b), are shown in figure 4.

space and is represented on a grid of $N_{\mathrm{p}}$ equally spaced values of internuclear distances by a $N_{\mathrm{p}} \times N_{\mathrm{p}}$ diagonal matrix. The kinetic energy operator, being diagonal in momentum space, is analytically Fourier transformed to a coordinate representation and is expressed by a nondiagonal matrix on the same grid. The diagonalization of the entire $N_{\mathrm{p}} \times N_{\mathrm{p}}$ Hamiltonian matrix yields eigenvalues and eigenvectors corresponding either to bound states or to (discretized) continuum states. The wavefunctions are represented by their values on a grid. The method is very convenient due to its global character and all eigenvalues are deduced from a single diagonalization. A grid with $N_{\mathrm{p}}=850$ points has been used, ranging from $4 a_{0}$ to $30 a_{0}$, which allows us to represent kinetic energy up to $24000 \mathrm{~cm}^{-1}$. We have chosen such a big grid to represent quite high kinetic energies, in order to take into account large portions of the dissociation continuum. Transition dipole moments are represented on the same grid and the transition matrix elements $\left\langle v^{\prime \prime}|D| v^{\prime}\right\rangle$ are computed by a quadrature formula where the grid points become sampling points. Finally, the contributions to continuum are properly normalized using the calculated density of states.

In figure 3(a) we present our quantum-mechanical simulations for the $1{ }^{3} \Sigma^{+}\left(v^{\prime \prime}=0\right)-N$ ${ }^{3} \Sigma^{+}\left(v^{\prime}\right)(N=2,3,4,5)$ transitions. As can be judged from figure 2(a) the most dominant feature is the $1^{3} \Sigma^{+}\left(v^{\prime \prime}=0\right)-2{ }^{3} \Sigma^{+}\left(v^{\prime}\right)$ progression (please note the logarithmic scale which emphasizes the onset of the series and far wing asymmetry). The avoided crossing between 3 ${ }^{3} \Sigma^{+}$and $4{ }^{3} \Sigma^{+}$states corresponds to ${ }^{3} \Sigma_{\mathrm{g}}^{+}$and ${ }^{3} \Sigma_{\mathrm{u}}^{+}$states in homonuclear dimer and the higher energy transition is more allowed than the lower one. The contribution of the bound-free continuum of the $1^{3} \Sigma^{+}-3{ }^{3} \Sigma^{+}$transition which is not well resolved is replotted on a larger scale in figure 4.

Figure 3(b) shows simulations for the $1^{3} \Sigma^{+}\left(v^{\prime \prime}=0\right)-N^{3} \Pi\left(v^{\prime}\right)(N=1,2,3,4)$ transitions. The most dominant feature is the $1{ }^{3} \Sigma^{+}\left(v^{\prime \prime}=0\right)-2{ }^{3} \Pi\left(v^{\prime}\right)$ band (see figure 2(c)). The needlelike shape of the band reflects the transitions near the flat local maximum of the upper state 


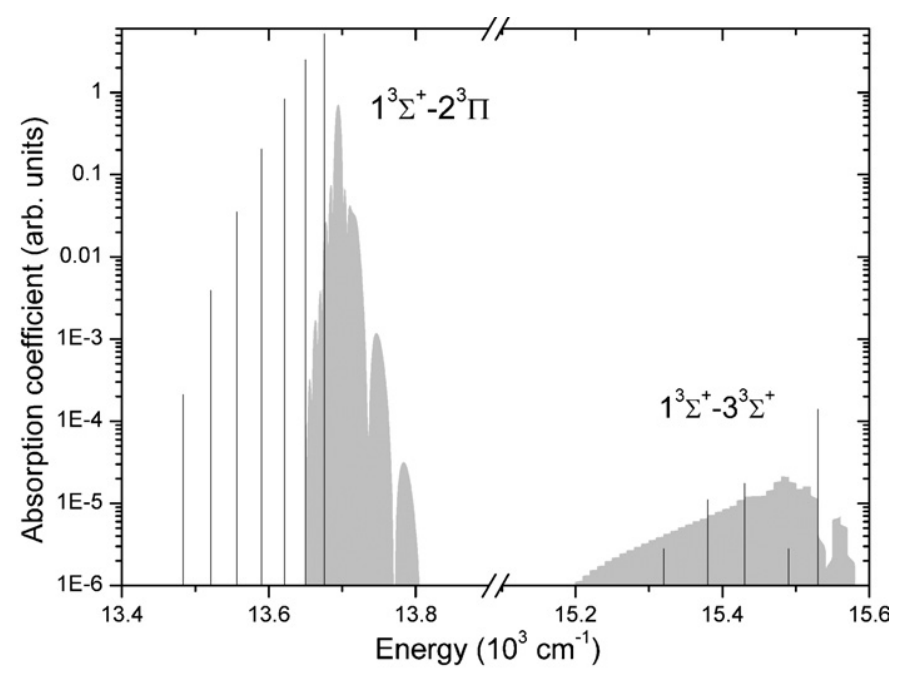

Figure 4. Bound-bound (vertical black lines) and bound-free (grey areas) contributions for 1 ${ }^{3} \Sigma^{+}-2{ }^{3} \Pi$ and $1{ }^{3} \Sigma^{+}-3{ }^{3} \Sigma^{+}$transitions.

(see figure 1(a)). The contribution of the bound-free continuum which is not well resolved is replotted on a larger scale in figure 4. The peculiar shape of the upper state is imprinted in the form of the $1^{3} \Sigma^{+}\left(v^{\prime \prime}=0\right)-3{ }^{3} \Pi\left(v^{\prime}\right)$ progression.

Quantum-mechanical simulations for singlet transitions are given in figures 3(c) and (d). The most dominant band among $1^{1} \Sigma^{+}\left(v^{\prime \prime}=0\right)-N^{1} \Sigma^{+}\left(v^{\prime}\right)(N=2,3,4,5)$ transitions is the $1{ }^{1} \Sigma^{+}\left(v^{\prime \prime}=0\right)-2{ }^{1} \Sigma^{+}\left(v^{\prime}\right)$ one. Because the corresponding transition dipole moment (see figure 2(b)) passes through zero, the very weak $1{ }^{1} \Sigma^{+}\left(v^{\prime \prime}=0\right)-5{ }^{1} \Sigma^{+}\left(v^{\prime}\right)$ band appears as a doublet.

Figure 3(d) displays $1^{1} \Sigma^{+}\left(v^{\prime \prime}=0\right)-N^{1} \Pi\left(v^{\prime}\right)(N=1,2,3,4)$ transitions. The first two bands correspond to the transitions to the weakly coupled 1 and $2^{1} \Pi$ 'twin' states which exhibit an avoided crossing resulting in a rapid change of electric dipole transition moments (see figure 2(d)) and much stronger intensity of the first band. The abrupt fall off of the second vibrational progression corresponds to the hump in the upper potential involved (see figure 1(b)). The contribution of the continuum states above the hump is negligible. Kasahara et al [24] observed the $1^{1} \Pi$ and $2{ }^{1} \Pi$ states using Doppler-free optical-optical double resonance polarization spectroscopy and derived molecular constants for these states assuming a particular vibrational numbering. The molecular constants $T_{\mathrm{e}}, \omega_{\mathrm{e}}$ and $R_{\mathrm{e}}$ of the $1{ }^{1} \Pi$ state were determined to be $15012.493 \mathrm{~cm}^{-1}, 61.256 \mathrm{~cm}^{-1}$ and $4.3813 \AA$, respectively. From our ab initio calculations we obtained $T_{\mathrm{e}}=15073.49 \mathrm{~cm}^{-1}, \omega_{\mathrm{e}}=59.52 \mathrm{~cm}^{-1}, R_{\mathrm{e}}=4.37 \AA$, whereas Leininger et al [22], for example, reported $T_{\mathrm{e}}=14973.4 \mathrm{~cm}^{-1}, \omega_{\mathrm{e}}=60.40 \mathrm{~cm}^{-1}$ and $R_{\mathrm{e}}=4.3919 \AA$. As for the $2{ }^{1} \Pi$ state, Kasahara et al [24] determined $T_{\mathrm{e}}=16210.252 \mathrm{~cm}^{-1}$, $\omega_{\mathrm{e}}=51.70 \mathrm{~cm}^{-1}$ and $R_{\mathrm{e}}=5.0822 \AA$. The spectroscopic observation of Amiot et al [25] led to the reassignment by 6 quanta of the vibrational numbering for the $2{ }^{1} \Pi$ state reported in [24] and the new recommended values are $T_{\mathrm{e}}=15902.06 \mathrm{~cm}^{-1}, \omega_{\mathrm{e}}=49.76 \mathrm{~cm}^{-1}$ and $R_{\mathrm{e}}=$ $5.088 \AA$ A. From our ab initio calculations we obtained $T_{\mathrm{e}}=15854.89 \mathrm{~cm}^{-1}, \omega_{\mathrm{e}}=52.93 \mathrm{~cm}^{-1}$, $R_{\mathrm{e}}=5.03 \AA$, whereas Leininger et al [22] reported $T_{\mathrm{e}}=15645.2 \mathrm{~cm}^{-1}, \omega_{\mathrm{e}}=63.48 \mathrm{~cm}^{-1}$ and $R_{\mathrm{e}}=5.1336 \AA$.

The spin-forbidden electric dipole $3{ }^{3} \Sigma^{+}-1{ }^{1} \Sigma^{+}$transitions observed by Lee et al [13] in the spectral region between 18600 and $19200 \mathrm{~cm}^{-1}$ show comparable intensities to the 
weak electric quadrupole $1{ }^{1} \Delta-1{ }^{1} \Sigma^{+}$transitions and according to our predictions appear to overlap with the much stronger electric dipole $1^{3} \Sigma^{+}\left(v^{\prime \prime}=0\right)-4{ }^{3} \Pi\left(v^{\prime}\right)$ band (see figure 3(b)). Therefore, we do not expect that it could be visible in experiment. Some of the peaks within the triplet manifold would exhibit the fine structure splitting due to spinorbit effects. The intensity pattern of absorption spectra in the gas phase shows a triplet peak structure which is not always the case in the observed excitation spectra of alkali dimers formed on helium nanodroplets as discussed in [8]. The precise intensity pattern depends on the shape of the interaction potentials (including the $R$-dependent spin-orbit splitting) and variation of transition moments, and in the case of dimers formed on helium nanodroplets on the additional coupling between the molecule and helium atoms within the cluster. We have not included these effects in the simulation. According to the comparison of well-known alkali dimer spectra in free gas phase and on helium nanodroplets [17] the helium-induced shift to the vibrational bands is not more than $5 \mathrm{~cm}^{-1}$, whereas the helium-induced broadening can be between 30 and $50 \mathrm{~cm}^{-1}$. Qualitatively, the shift can be explained by the changes of radiative properties of the atoms (molecules) placed close to a dielectric surface and the broadening as being due to interactions of trapped atoms with the surface and bulk excitations of the helium clusters, but (at least for the atoms) mainly due to the fact that excitation predominantly occurs into the repulsive part of the atom-cluster potential energy surface [26].

In figure 4 the spectral simulations of the $1^{3} \Sigma^{+}-2{ }^{3} \Pi$ and $1{ }^{3} \Sigma^{+}-3{ }^{3} \Sigma^{+}$transitions are replotted on a larger scale, clearly displaying the contributions of the bound-free continua which are not well resolved in figures 3(b) and (a), respectively. The broad maximum of the $2{ }^{3} \Pi$ potential curve lies right in the middle of the Franck-Condon window and transitions to the bound states are basically extended into the continuum which exhibits some additional structure. The $3{ }^{3} \Sigma^{+}$potential curve is repulsive within the Franck-Condon window reaching the maximum at the lower end. The repulsive branch leads to the continuum spectrum and the superimposed discrete lines correspond to the quasibound states (resonances) in the inner well of the $3{ }^{3} \Sigma^{+}$potential.

As already mentioned in section 1, laser spectroscopic experiments were carried out for $\mathrm{KRb}$ molecules in magneto-optical traps with potassium-rubidium mixtures [6, 7] where only near-dissociation spectra have been observed, probing the long-range parts of interaction potentials. Transitions from the $v^{\prime \prime}=86-92$ levels of the $\mathrm{X}$ state, which are bound by up to $30 \mathrm{~cm}^{-1}$, and the $v^{\prime \prime}=17-23$ levels of the a state, which are also bound by up to $30 \mathrm{~cm}^{-1}$, were observed. The analysis was based on the potential curves from [10] and the measured vibrational spacings were found to be in excellent agreement with those previously measured and those calculated from the relevant potential curves. We do not expect that new simulations based on our present $a b$ initio potential curves (which are of the same overall quality as those from [10]) would improve the knowledge acquired in [6, 7].

\subsection{Absorption at high temperatures}

A simple absorption experiment similar to those described earlier [16, 27] was performed. A 1:1 mixture of rubidium and potassium was contained in a cylindrical, linear all-sapphire cell (ASC) with an inner diameter of $10 \mathrm{~mm}$ and a length of $160 \mathrm{~mm}$. Due to the special procedures in manufacturing the cell, the cell can be heated up to $700{ }^{\circ} \mathrm{C}$. In order to prevent metal vapour deposition on the windows of the cell, the cell was placed in an oven equipped with three separate heaters. Above the heated liquid, mixed metal vapour consists predominantly of rubidium and potassium atoms, but there is also a small percentage of diatomic molecules, $\mathrm{Rb}_{2}, \mathrm{KRb}$ and $\mathrm{K}_{2}$ (in the decreasing order of densities). The cell was irradiated by the continuous light from a halogen lamp. The transmitted light was collected and focused onto 


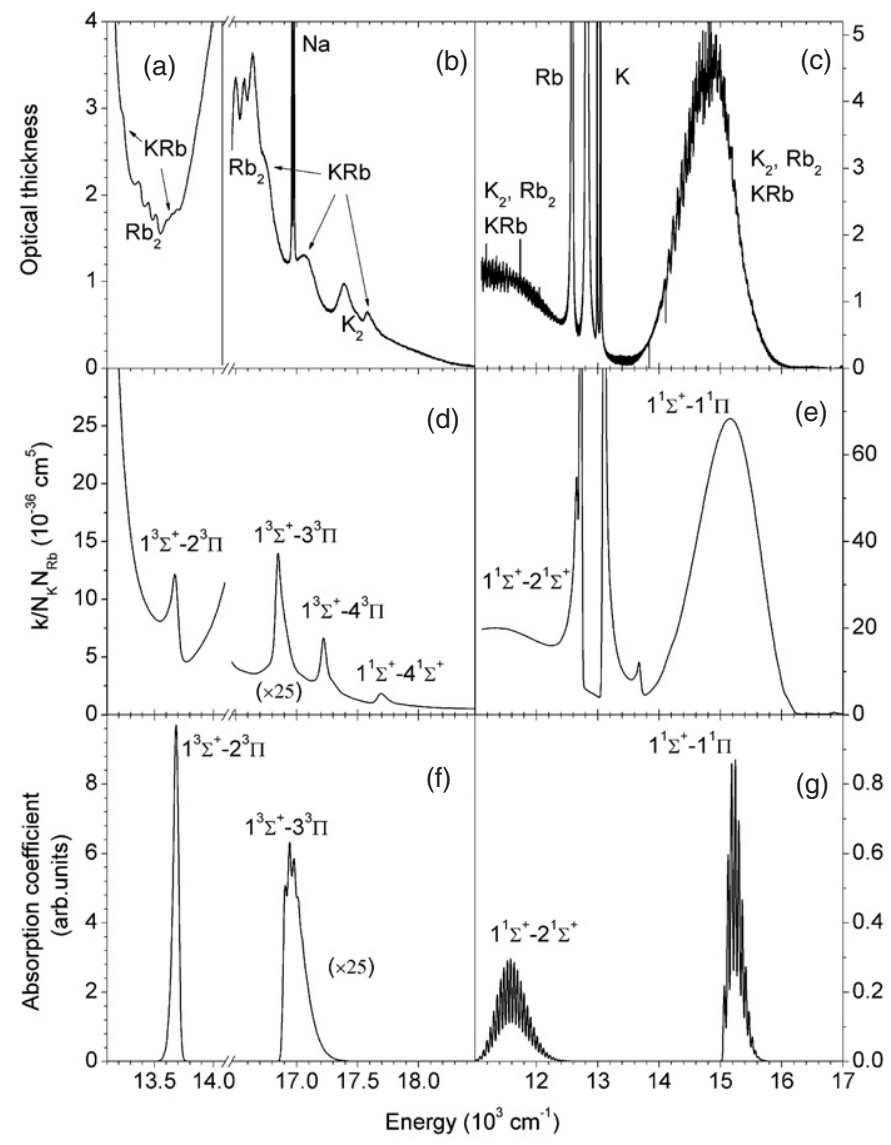

Figure 5. Absorption spectrum of a potassium-rubidium mixture in an all-sapphire cell obtained with a high-resolution scanning monochromator at three different temperatures: (a) $613 \mathrm{~K}$, (b) $713 \mathrm{~K}$, (c) $575 \mathrm{~K}$; (d) and (e) semiclassical simulation of the high-temperature reduced absorption coefficient at $650 \mathrm{~K}$ for the spectral region shown in (a)-(c); (f) and (g) prediction of absorption bands of $\mathrm{KRb}$ under low-temperature conditions for triplet (f) and singlet ( $\mathrm{g}$ ) transitions. In figures (d) and (f) the traces have been multiplied by a factor of 25 for easier visibility of the spectra.

the entrance slit of a $1.5 \mathrm{~m}$ Jobin-Yvon THR-1500 scanning monochromator (1200 grooves $\mathrm{mm}^{-1}$ holographic grating) with a spectral resolution of $0.015 \mathrm{~nm}$. Spectrally resolved light was detected with a Hamamatsu R936 photomultiplier placed at the exit slit of the monochromator. The electrical signal was amplified by a Stanford Research Systems SR510 lock-in amplifier (connected to a SR560 mechanical light chopper) and fed to a laboratory computer.

Absorption measurements were performed in the spectral region from $300 \mathrm{~nm}$ to $900 \mathrm{~nm}$, determined by the sensitivity of the detection system. In this wide spectral region different atomic and homo- and heteronuclear molecular features appeared in the absorption spectra depending on the temperature of the mixed vapour. The absorption coefficient of the $\mathrm{K}+\mathrm{Rb}$ mixed vapour at temperature $T$ was obtained experimentally from the Beer-Lambert law [28].

In figures 5(a)-(c) we show the optical thickness of the hot $\mathrm{K}-\mathrm{Rb}$ vapour mixture derived from a measured transmission spectrum. In a hot $\mathrm{K}-\mathrm{Rb}$ vapour mixture spectral signatures of both $\mathrm{K}_{2}$ and $\mathrm{Rb}_{2}$ dimers appear together with $\mathrm{KRb}$ bands. Different $\mathrm{KRb}$ bands (depending upon the molecular states responsible for the formation of the band) appear in 
the absorption spectra at different temperatures. In order to stress all KRb molecular features in the measured spectral range the presented optical thickness is divided into three spectral regions corresponding to three different vapour temperatures.

In the spectral region shown in figure 5(a), well-known $\mathrm{Rb}_{2}$ triplet satellite bands $\left(1^{3} \Sigma_{\mathrm{u}}^{+} \rightarrow 1^{3} \Pi_{\mathrm{g}}\left(\Omega=0_{\mathrm{g}}^{+}, 0_{\mathrm{g}}^{-}, 1_{\mathrm{g}}, 2_{\mathrm{g}}\right)\right.$ transitions $)$ can be observed [29] with the main peaks at 13367,13439 and $13517 \mathrm{~cm}^{-1}$. $(\Omega=\Lambda+\Sigma$ is the projection of the total electronic angular momentum onto the molecular axis, whereas $\Lambda$ and $\Sigma$ are projections of the total electronic orbital angular momentum and the total electron spin, respectively.) The small undulation in the blue wing of the potassium resonant line at $13236 \mathrm{~cm}^{-1}$ is a satellite band of the long-range triplet transition $1^{3} \Sigma^{+} \rightarrow 2^{3} \Pi\left(0^{+}\right)$in the KRb molecule [16]. KRb triplet satellite bands with peaks at $13611,13659,13697 \mathrm{~cm}^{-1}$ are results of $1^{3} \Sigma^{+} \rightarrow 2^{3} \Pi\left(0^{+}, 0^{-}\right.$, 1, 2) transitions [16].

Figure 5(b) comprises the spectral region of diffuse bands. $\mathrm{Rb}_{2}$ (maxima at 16518,16578 and $16647 \mathrm{~cm}^{-1}$ ) and $\mathrm{K}_{2}$ (maximum at $17391 \mathrm{~cm}^{-1}$ ) diffuse bands are identified as $1^{3} \Sigma_{\mathrm{u}}^{+} \rightarrow$ $2{ }^{3} \Pi_{\mathrm{g}}$ free-free and bound-free transitions and they are well known in the literature [30]. In the same spectral region, there are three distinct KRb diffuse bands at 16750, 17044 and $17575 \mathrm{~cm}^{-1}[15]$.

In figure 5(c) the red part of the absorption spectra ranging from 11000 to $17000 \mathrm{~cm}^{-1}$ is shown. In this spectral range the $\mathrm{K}_{2}$ and $\mathrm{Rb}_{2}[31,32]$ and $\mathrm{KRb}$ molecular bands (traditionally denoted by $\mathrm{X}-\mathrm{A}$ and $\mathrm{X}-\mathrm{B}$, respectively) are observed besides the $\mathrm{K}$ and $\mathrm{Rb}$ atomic first resonance lines. Due to the very similar pattern of interaction potentials and relatively high vapour temperature, $\mathrm{K}_{2}, \mathrm{Rb}_{2}$ and $\mathrm{KRb}$ molecular bands of the same symmetry overlap resulting in two broad bands.

In figures 5(d) and (e) we present a semiclassical simulation of the reduced absorption coefficient of the KRb molecule for the temperature of $650 \mathrm{~K}$. This temperature was chosen as an average of temperatures in experiments presented in figures 5(a)-(c). The maximum intensity of the satellite band formed by the $1^{3} \Sigma^{+} \rightarrow 2^{3} \Pi$ transition (figure $5(\mathrm{~d})$ ) at $13675 \mathrm{~cm}^{-1}$ is in a good agreement with the shape and position of the triplet satellite band middle peak (figure 5(a)). The shift from the theoretical peak is only $16 \mathrm{~cm}^{-1}$. The peak intensity of the $1^{3} \Sigma^{+} \rightarrow 3{ }^{3} \Pi$ transition lies at $16847 \mathrm{~cm}^{-1}$ which is shifted by $97 \mathrm{~cm}^{-1}$ from the position of the experimental counterpart in figure 5(b). The next triplet transition $1^{3} \Sigma^{+}$ $\rightarrow 4^{3} \Pi$ with an absorption maximum at $17224 \mathrm{~cm}^{-1}$ is shifted by $180 \mathrm{~cm}^{-1}$ compared to the experimental position. The singlet transition $1{ }^{1} \Sigma^{+}-4{ }^{1} \Sigma^{+}$with peak intensity at $17693 \mathrm{~cm}^{-1}$ is shifted from the experiment by $118 \mathrm{~cm}^{-1}$. Differences between positions of absorption maxima in experimental and simulated spectra are well within the standard accuracy of computed transition energies. In figure 5(e) two broad absorption features are shown. One is related to the $1{ }^{1} \Sigma^{+} \rightarrow 2{ }^{1} \Sigma^{+}$transition and corresponds to the $\mathrm{X}-\mathrm{A}$ band. At higher photon energies, the calculated $1^{1} \Sigma^{+} \rightarrow 1^{1} \Pi$ transition describes the $\mathrm{X}-\mathrm{B}$ band (figure $5(\mathrm{c})$ ). Note, however, that in the actual experiment the $\mathrm{KRb}$ bands overlap with the $\mathrm{K}_{2}$ and $\mathrm{Rb}_{2}$ bands.

Figures $5(\mathrm{f})$ and $(\mathrm{g})$ show our simulations of the most prominent bands which should be observable in an excitation spectrum of a $\mathrm{KRb}$ diatomic attached to a cold helium droplet. While the interaction of the alkali atoms and molecules with helium is not explicitly included in the energy potentials, our spectral simulations are accounting for the expected broadening effects through helium by convoluting the calculated bands with a Gaussian of $40 \mathrm{~cm}^{-1}$ width. Together with the fact that we show only excitations from the lowest vibrational state of $1{ }^{1} \Sigma^{+}$ and $1{ }^{3} \Sigma^{+}$due to the helium temperature of $0.37 \mathrm{~K}$, the predictions can provide an important step for the identification of recorded alkali spectra on helium droplets with regard to relative intensities and widths of the band envelopes. A good example in this respect has been the recent assignments of caesium molecular bands [33]. 
The $2{ }^{3} \Pi-1{ }^{3} \Sigma^{+}$transition was identified both in absorption and emission [8] and the predictions of this paper provided a guideline for the assignment of spectra.

As we work with Hund's case (a) potentials, fine structure effects have not been considered so far but could be included by applying the relevant spin-orbit matrix elements. However, for a first comparison with alkali dimer spectra on helium, the inclusion of the fine structure may not help because the experimental observations indicate some strong deviation from the regular spin-orbit splittings in free molecules [8]. Instead of three bands as expected for a gas-phase spectrum, Allard et al [8] obtained two broad peaks with no resolved vibrational structure. In a thorough analysis of the absorption and emission spectra they used the same Hund's case (a) potential curves and transition dipole moments as we have used in the present work. Further details about a test of the accuracy of the present $a b$ initio potentials can be found in [8].

Generally, the absorption of a KRb molecule sitting on a cold He droplet does not have a direct resemblance with the absorption of the dense vapour containing some KRb molecules, since in the later case all populated vibrational states have to be accounted for in the simulated spectra. However, the singlet band progressions deriving from $1^{1} \Sigma^{+}\left(v^{\prime \prime}=0\right)$ can be seen as seeds for the bands formed at elevated temperatures (see figures 5(c), (e) and (g)). The low temperature absorption coefficient simulations for $1^{1} \Sigma^{+} \rightarrow 2{ }^{1} \Sigma^{+}$and $1{ }^{1} \Sigma^{+} \rightarrow 1{ }^{1} \Pi$ (figure 5(g)) transitions have peak intensities at $11433 \mathrm{~cm}^{-1}$ and $15144 \mathrm{~cm}^{-1}$, respectively. This coincides with the high temperature counterparts in 5(e). As for triplet transitions, one can see that two bands shown in figure 5(f) correspond to two features observed in thermal vapours shown in figures 5(a), (b) and (d). From figure 1 it is obvious that both upper state potentials in question are rather shallow in the Franck-Condon regime, and within the simple quasistatic picture one can expect satellite bands in the vicinity of a classical singularity that corresponds to an extremum in the difference potential curve.

The position and shape of calculated excitation bands depend critically on the accuracy of the calculated potentials used. A comparison of our theoretical simulation with experimentally observed spectra of $\mathrm{KRb}$ in absorption in dense hot vapours provides an opportunity to estimate the accuracy of the difference potentials around the ground-state potential minimum. If desired, one might slightly adapt the potential curves and accordingly shift the peak positions in simulated low-temperature absorption spectra. Transitions between triplet states of the homonuclear dimers $\mathrm{Rb}_{2}[34]$ and $\mathrm{Cs}_{2}$ [30] as well as the heteronuclear LiCs [35] on helium nanodroplets have recently been published. The $1^{3} \Sigma^{+}-2{ }^{3} \Pi$ transition in KRb have recently been identified in absorption on cold helium droplets [8] and more bands will hopefully be identified with the help of our predictions.

\section{Conclusion}

We have predicted absorption bands of $\mathrm{KRb}$ molecules by using ab initio potential curves in Hund's case (a) and taking into account recently calculated transition dipole moments. The described results correspond to the experimental situation in which KRb molecules are excited from their vibronic ground state (either in the singlet or in the triplet potential), as it would be the case for example on the surface of cold helium clusters. They may serve as a guide in future experiments for comparison purposes. In addition to this, we discussed the absorption spectra of dense $\mathrm{K}-\mathrm{Rb}$ vapour at high temperatures, since triplet states are populated under these conditions as well. Comparing a semiclassical simulation based on the same $a b$ initio calculation and our experimental hot vapour observations, we obtained the long-sought interpretation of three $\mathrm{KRb}$ diffuse bands in the broad spectral region around $600 \mathrm{~nm}$. We have found shifts between calculated and observed band positions which indicate 
the limits of accuracy of the used potentials. This has to be kept in mind when absorption spectra of alkali mixtures on cold helium droplets have to be assigned. In the visible and nearinfrared spectral region we predicted two strong triplet transitions with their peak intensities at $13653 \mathrm{~cm}^{-1}\left(1^{3} \Sigma^{+} \rightarrow 2^{3} \Pi\right)$ and $16890 \mathrm{~cm}^{-1}\left(1^{3} \Sigma^{+} \rightarrow 3^{3} \Pi\right)$ and two stronger singlet transitions with peak intensities at $11433 \mathrm{~cm}^{-1}\left(1^{1} \Sigma^{+} \rightarrow 2{ }^{1} \Sigma^{+}\right)$and $15144 \mathrm{~cm}^{-1}\left(1^{1} \Sigma^{+} \rightarrow\right.$ $\left.1^{1} \Pi\right)$. So far, only the first of these four has been identified on helium droplets [8].

\section{Acknowledgments}

We acknowledge the support from the Ministry of Science and Technology of the Republic of Croatia (projects 0035002 and 0035004), the European Commission Research Training Network on 'Cold Molecules' (FW 5). The Austrian-Croatian bilateral project no. 3/2004 and the French-Croatian bilateral project COGITO enabled support for fruitful mutual visits.

\section{References}

[1] Bevan P V 1910 Proc. R. Soc. A 83421

[2] Walter J M and Barratt S 1928 Proc. R. Soc. A 105221

[3] Fioretti A, Comparat D, Crubellier A, Dulieu O, Masnou-Seeuws F and Pillet P 1998 Phys. Rev. Lett. 804402

[4] Bethlem H L, Berden G and Meijer G 1999 Phys. Rev. Lett. 831558

[5] Mancini M W, Telles G D, Caires A R L, Bagnato V S and Marcassa L G 2004 Phys. Rev. Lett. 92133203

[6] Wang D, Qi J, Stone M F, Nikolayeva O, Wang H, Hattaway B, Gensemer S D, Gould P L, Eyler E E and Stwalley W C 2004 Phys. Rev. Lett. 93243005

[7] Wang D, Eyler E E, Gould P L and Stwalley W C 2005 Phys. Rev. A 72032502

[8] Allard O, Nagl J, Auböck G, Callegari C and Ernst W E 2006 J. Phys. B: At. Mol. Opt. Phys. 39 S1169

[9] Park S J, Choi Y J, Lee Y S and Jeung G-H 2000 Chem. Phys. 257135

[10] Rousseau S, Allouche A R and Aubert-Frécon M 2000 J. Mol. Spectrosc. 203235

[11] Kotochigova S, Julienne P S and Tiesinga E 2003 Phys. Rev. A 68022501

[12] Amiot C 2000 J. Mol. Spectrosc. 203126

[13] Lee Y, Yun Ch, Yoon Y, Kim T and Kim B 2001 J. Chem. Phys. 1157413

[14] Lee Y, Yoon Y, Kim B, Li L and Lee S 2004 J. Chem. Phys. 1206551

[15] Beuc R, Milosevic S and Pichler G 1984 J. Phys. B: At. Mol. Phys. 17739

[16] Skenderović H, Beuc R, Ban T and Pichler G 2002 Eur. Phys. J. D 1949

[17] Higgins J, Callegari C, Reho J, Stienkemeier F, Ernst W E, Gutowski M and Scoles G 1998 J. Phys. Chem. A 1024952

[18] Aymar M and Dulieu O 2005 J. Chem. Phys. 122204302

[19] Huron B, Malrieu J-P and Rancurel P 1973 J. Chem. Phys. 585745

[20] Durand P and Barthelat C 1975 Theor. Chim. Acta 38283

[21] Foucrault M, Millié Ph and Daudey J P 1992 J. Chem. Phys. 961257

[22] Leininger T, Stoll H and Jeung G-H 1997 J. Chem. Phys. 1062541

[23] Meyer R 1970 J. Chem. Phys. 522053

[24] Kasahara S, Fujiwara C, Okada N, Kato H and Baba M 1999 J. Chem. Phys. 1118857

[25] Amiot C, Vergès J, Effantin C and d'Incan J 2000 Chem. Phys. Lett. 32121

[26] Stienkenmeier F, Higgins J, Callegari C, Kanorsky S I, Ernst W E and Scoles G 1996 Z. F. Phys. D 38253

[27] Aumiler D, Ban T, Beuc R and Pichler G 2003 Appl. Phys. B 76859

[28] Demtröder W 2003 Laser Spectroscopy: Basic Concepts and Instrumentation (New York: Springer) p 441

[29] Awann H S and Lewis E L 1976 J. Phys. B: At. Mol. Phys. 9 L551

[30] Pichler G, Milošević S, Veža D and Beuc R 1983 J. Phys. B: At. Mol. Phys. 164619

[31] Amiot C, Dulieu O and Vergés J 1999 Phys. Rev. Lett. 832316

[32] Amiot C and Vergés J 1997 Chem. Phys. Lett. 27491

[33] Ernst W E, Huber R, Jiang S, Beuc R, Movre M and Pichler G 2006 J. Chem. Phys. 124024313

[34] Brühl F R, Miron R A and Ernst W E 2001 J. Chem. Phys. 11510275

[35] Mudrich M, Bünermann O, Stienkemeier F, Dulieu O and Weidemüller M 2004 Eur. Phys. J. D 31291 